\title{
Sperm numbers and fertility: a kinetic approach
}

\author{
C. VAN DUIJN Jnr. \\ Biophysics Department, Research Institute for Animal Husbandry "Schoonoord", \\ Zeist, Netherlands
}

\section{Summary}

A simple kinetic theory is derived for the relationship between numbers of spermatozoa, their movement, and the probability of fertilization; the theory shows good agreement with experimental results.

Fertility $F$ (expressed in any convenient measure such as "\% non-returns" as is usual in A.I. practice) is shown to be related to the number of normally moving spermatozoa $N_{0}$ shed or inseminated at $t_{o}$ at some distance from the ovum, with a mean swimming velocity $\bar{v}_{o}$, by:

$$
F=F_{\infty}\left\{1-\exp \left[-\frac{\mathrm{k}_{F}}{\mathrm{k}_{N_{v}}} \cdot \ln \left(\frac{N_{0} \cdot \bar{v}_{0}}{N_{i} \cdot \overline{v_{i}}}\right)\right]\right\}
$$

where $F_{\infty}=$ maximal value of fertility attainable with the given population of spermatozoa and females (computed from experimental data); $N_{i}$ and $\bar{v}_{i}$ are the minimum number of moving spermatozoa with minimum mean velocity required to let the next spermatozoon make the chance of fertilization greater than zero, the mathematical minimum requirements being $N_{i}+1 \geqslant 1, \bar{v}_{i}>0$. The constants $\mathrm{k}_{F}$ and $\mathrm{k}_{N v}$ are the rate constants corresponding to the two assumptions on which the theory is based: (i) The numbers of spermatozoa that can fertilize an ovum decrease exponentially with time; (ii) The rate of increase of probability of fertilization changes exponentially with time. The results are in agreement with published experimental information.

There is a linear relationship between $\log F$ and $t_{1 / 2}(N \bar{v}) \cdot \log (N \bar{v})_{0}$. The validity of this criterion has been tested by correlating it with field data of fertilizations obtained in artificial insemination of cows. The predicted linear functional relationships were obtained with correlation coefficients of $r=0.98$ during the grazing period, and $r=0.995$ during the housing period $(P \ll 0.001)$ over a 4-year period with 184,310 inseminations, whereas correlations between fertility and the separate variables in the same material were all insignificant.

\section{Introduction}

The relationship between the number of spermatozoa and the probability that fertilization will occur is important both with respect to evaluation of semen quality and fertility in general and, in artificial insemination (A.I.), for determining the most efficient dilution rates. Many investigations have been performed, surveys having been given by ANDERSON (1945) for animals and by FARRIS (1950) for man.

Received for publication 25th September, 1964. 
It is generally agreed that at low sperm densities fertilization probability increases with increasing numbers up to a certain optimum, after which only a slight or no further increase is obtained (Branton, James, Patrick and Newsom, 1951; Branton, Kellgren and Patrick, 1953; Lasley and Bogart, 1943 ; Herman and Swanson, 1951; Mercier and Salisbury, 1946; Stone, Johnston and Mixner, 1950; Swanson and Herman, 1947 (these refer to cattle); Plisko, 1961 (pigs); Taneja and Gowe, 1961, 1962 (fowl)).

So far, empirical results have not been evaluated further than by application of some simple statistics, indicating at least that the relationship between fertility $F$ and total numbers of spermatozoa per unit volume, $N$, deviates significantly from linearity (Branton, James, Patrick and Newsom, 1951; Bratton, Foote and Henderson, 1954; WiLletT and LARSON, 1952).

Branton, Kellgren and Patrick (1953) considered that numbers of moving spermatozoa would be a more adequate parameter than total number of spermatozoa. They therefore multiplied the latter (determined with very good accuracy from measurements of light transmission) by visual estimates of percentage motility, which are subject to large errors. Whereas the original estimates using total numbers deviated significantly from linearity, significance was lost after multiplication by the motility estimates, from which the authors concluded that the relationship was in reality linear, which is wrong because they did not consider the fundamental boundary condition, that at sperm concentration 0 , fertility must be 0 , whereas their regression showed about $50 \%$ fertility when the number of motile spermatozoa, $N m(N=$ total number, $m=\%$ motility rating), was zero. Further, they stated that "statistical analysis revealed that there was no significant difference between results with 6 and $12 \times 10^{8}$ spermatozoa per $\mathrm{ml}(\mathrm{P}<0.05)$. However, $2 \times 10^{6}$ gave significantly lower fertility than A or B $(P<0.01)$ ".

This information shows that, contrary to their interpretation, there is a significant deviation from linearity, because for $N m=6-12 \times 10^{6} / \mathrm{ml}, \mathrm{d} F / \mathrm{d} N \rightarrow 0$, but for $N m=2-6 \times 10^{6} / \mathrm{ml}, \mathrm{d} F / \mathrm{d} N>0$. There is, therefore, a changing gradient over the curve whereas linearity requires that $\mathrm{d} F / \mathrm{d} N=$ const. over the entire range. This argument, combined with the boundary condition just mentioned, shows that any disagreement between the conclusions of BRANTON, KellgReN and PATRICK (1953) with the earlier results of Branton, James, Patrick and Newsom (1951) is not due to the actual results, but to misinterpretation. It will be shown that the 1953 results are in agreement with the theory presented in this paper.

A functional relationship between sperm numbers, motility parameters and fertility has been derived from simple kinetic considerations. The results have been checked by application to published experimental information and to my own material, with good agreement.

\section{Theory}

In the most general case spermatozoa are produced and deposited at a distance from the ovum and must be transported either actively or passively, which takes time.

First assumption

The number of spermatozoa capable of fertilizing an ovum decreases exponentially with time, environmental conditions being constant, according to:

$$
N(\mathrm{t})=N_{0} \cdot \mathrm{e}^{-\mathrm{k}_{N} \cdot \mathrm{t}}
$$

Neth. J. agric. Sci., Vol. 13 (1965) No. 4 (November) 
where $N(\mathrm{t})=$ number of fertile spermatozoa available at time $t, N_{0}=$ the initial number shed or inseminated at time $t_{o}$ at a distance $\triangle p$ from the meeting place with the ovum.

This assumption is consistent with experimental evidence (RIKMENSPoel, 1957, 1960; RikMENSPOEl and vaN Duisn, 1960; van DuUn and RIKMENSPOEL, 1960).

\section{Second assumption}

The rate of increase of probability of fertilization changes exponentially with time.

Let a number $a$ of spermatozoa, just sufficient to make the probability of fertilization $>0$ approach an ovum in each time inverval $\delta t$, during which no deterioration of the spermatozoa is assumed. Then the chance that fertilization will occur increases with time, but only up to the moment of fertilization, because an egg can only be fertilized once. Hence the increment of the probability of fertilization during successive time intervals after $t_{o}$ decreases asymptotically to zero. Now let $F$ denote the chance that during the last time interval $\delta t$ of a period ending at $t$, and during which period $t-t_{o}$ spermatozoa have been supplied at a constant rate, fertilization is accomplished. This chance increases (at a decreasing rate) with time up to a limiting value, governed by the probability that the ovum and the spermatozoa are fertile. Denoting by $F_{\infty}$ the maximum value that, with a particular sperm population and eggs of particular fertility, can be reached, the rate of change of $F$ is represented by:

$$
\frac{\mathrm{d} F}{\mathrm{~d} t}=\mathrm{k}_{F}\left(F_{\infty}-F\right)
$$

This equation is solved by separation of the variables and integrating between the moving limit $F$ corresponding with the variable $t$, and the fixed limit $F=0$ at $t=0$.

$$
\int_{F=0}^{F} \frac{\mathrm{d} F}{\left.F_{\mathrm{o}}-F\right)}=\mathrm{k}_{F} \int_{t=0}^{t} \mathrm{~d} t
$$

The integration yields:

$$
\ln \left(\frac{F_{\infty}}{F_{\infty}-F}\right)=\mathrm{k}_{F} \cdot t
$$

This equation thus gives the relationship between the probability of fertilization and the period of time during which spermatozoa are supplied at a constant rate. However, in reality spermatozoa will not be supplied at a constant rate, but at a decreasing rate according to the first assumption. Solving equation (1) for $t$ we get:

$$
t=\frac{1}{\mathrm{k}_{N}} \cdot \ln \frac{N_{o}}{N(\mathrm{t})}
$$

The time in equation (5) is the same as in (4) if we take $N_{0}$ to represent the number of spermatozoa deposited at once at $t=0$ at a distance from the ovum, and $N(\mathrm{t})=N_{i}$, if $N_{i}$ denotes the minimum number of spermatozoa that must be shed at this distance from the meeting place with the ovum to satisfy the conditions $F\left(N_{i}\right)=0, F\left(N_{i}+1\right)>0$. Since at least one spermatozoon must reach the ovum, 
the physical boundary condition is $N_{i}+1 \geqslant 1$. Substituting $t$ in equation (4) by (5) with the boundary condition just mentioned, we get:

$$
\ln \left(\frac{F_{\infty}}{F_{\infty}-F}\right)=\frac{\mathrm{k}_{F}}{\mathrm{k}_{N}} \cdot \ln \left(\frac{N_{0}}{N_{i}}\right)
$$

Equation (5) can be written in the exponential form and after rearrangement we get:

$$
F=F_{\infty}\left\{1-\exp \left[-\frac{\mathrm{k}_{F}}{\mathrm{k}_{N}} \cdot \ln \left(\frac{N_{o}}{N_{i}}\right)\right]\right\}
$$

which may also be written as:

$$
F=F_{\infty}\left\{1-\left(\frac{N_{0}}{N_{i}}\right)^{-\mathrm{k}_{F} / \mathrm{k}_{N}}\right\}
$$

The constants $F_{\infty}$ and $\mathrm{k}_{F}$ will be functions of all factors causally related to fertility in both males and females, other than sperm numbers, but, with respect to a given sperm population, they are really constant. $\mathbf{k}_{N}$ represents the rate constant (reciprocal of the characteristic time) of the decrease of numbers of spermatozoa capable of fertilizing an ovum; it is related to the concept of half-life periods $t_{1 / 2}(N)$ by $1 / k_{N}=$ $71 / 2(N) /$ In 2 , hence $t_{1 / 2}(N)=0.693 / \mathrm{k}_{N}$.

Since the actual number of spermatozoa that are capable of fertilizing an ovum (which thus far cannot be determined directly) can always be expressed as a fraction of the total number, equations (5) to (8) will apply irrespective of the way in which sperm numbers are being evaluated, the only effect being different numerical values of the sperm constants, especially $N_{i}$.

This can be used to advantage in studying practical fertility problems, since in such circumstances $N_{i}$ can be evaluated as an objective parameter for the fertilizing capacity of the spermatozoa in an ejaculate, in addition to $\mathrm{k}_{N}$ or $\mathrm{t}_{1 / 2}(N)$.

It should be emphasized that in this kinetic derivation no specific assumptions have been made with respect to the way in which sperm migration from the site of ejaculation to the ovum takes place, hence it appears that the equations must be valid in a most general sense, since they are independent of whether spermatozoa are transported actively by their own movement, or passively, or by an unknown combination of both transporting mechanisms.

An important implication of eq. (7) is that the half-life period of numbers of fertile spermatozoa is more important with respect to fertility than $N_{0}$ alone. The former may be determined from a series of live/dead estimations as a function of storage time under standardized conditions.

\section{Third assumption}

Active movement of spermatozoa is necessary

If this further assumption is made, that active sperm migration is a main mechanism (at least during the final part of the journey) involved in reaching the ovum (EDGAR and ASDELL, 1960), the theory may be extended to include velocity characteristics. Instead of the assumption contained in eq. (1) we get the rate of migration decreasing exponentially with time during the fertile period, which is also in agreement with 
experimental evidence (VAN DuiJn and RIKMENSPOel, 1960; van Duin, 1960). $N$ now representing the number of spermatozoa moving normally, with a mean velocity $\bar{v}$, we get:

$$
(N \cdot \bar{v})(\mathbf{t})=(N \cdot \bar{v})_{0} \cdot \mathrm{e}^{-\mathrm{k}_{N \nu} \cdot t}
$$

Eq. (2) remains unchanged. Since the only difference with respect to the previous condition is a substitution of $N \cdot \vec{v}$ for $N$ with the corresponding change of $\mathrm{k}_{N}$ to $\mathbf{k}_{N \nu}$ the further derivation follows the same way, resulting in:

$$
F=F_{\infty}\left\{1-\exp \left[-\frac{\mathrm{k}_{F}}{\mathrm{k}_{N \nu}} \cdot \ln \left(\frac{N_{0} \cdot \bar{v}_{0}}{N_{i} \cdot \bar{v}_{i}}\right)\right]\right\}
$$

or the corresponding analogue of eq. (7). Now $N \cdot \bar{v}$ is proportional to the number of spermatozoa passing by a certain spot in the field per unit time, as can be determined experimentally with RIKMENSPOEL's photo-electric apparatus (RIKMENSPOEL, 1957; Rikmenspoel and van Herpen, 1957; RikMENSPoel, van Herpen, van Dam and EiJKHOUT, 1960 ; VAN DUIJN, 1961) or by other convenient procedures, whereas the halflife period for the number of passages per unit time $t_{1 / 2}(N \bar{v})=0.693 / \mathbf{k}_{N}$, may be substituted for the rate constant $\mathrm{k}_{N_{v}}$, and $\mathrm{k}_{F}$ may be substituted by $\mathrm{t}_{1 / 2}(F)=$ $0.693 / \mathrm{k}_{F}$.

The differential form of eq. (10) is:

$$
\frac{\mathrm{d} F}{\mathrm{~d}(N \bar{\nu})}=\frac{\mathrm{k}_{F}\left(F_{\infty}-F\right)}{\mathrm{k}_{N_{v}} \cdot N \cdot \bar{\nu}}
$$

It can also be obtained by combining the three presumptions directly and integrated afterwards to yield eq. (10) instead of integrating the separate equations first and then combining the results, as has been done in the preceding derivation. Although the former was the actual procedure followed in the original derivation, the present step-by-step presentation was adopted because it appeared to allow more concise reasoning while making no difference whatsoever with respect to the mathematics involved.

\subsection{Derivation of parameters for semen evaluation}

Fundamental considerations: Spermatozoa are shed at a distance of space and time $\Delta(p, t)$ from the ovum, but now $t$ is made variable by allowing the egg not to be present when the required minimum number of spermatozoa arrives at $p$. The egg is produced at a random time; if it has been fertilized, no other one is produced; the ovum will degenerate with time if not fertilized, in which case, again, no new one will be produced. This applies in many mammals.

Minimum requirements for fertilization are now that at time $t$ when a fertilizable egg is produced, there are at least $N_{i}+\delta N$ viable spermatozoa in the field between $\triangle p$ and $p, \delta N$ having arrived at $\delta(p, t)$ from the ovum ( $\delta N$ is the smallest excess that makes fertilization possible). If active movement is required during the final period, we must substitute the migration rate $N \bar{v}$ for $N$, which does not change the shape of the function as set forth in the preceding part of this paper. 
Putting $N(\mathrm{t})=N_{i}$ in eq. (1), $t$ will represent the period during which the number of spermatozoa is sufficient to provide a positive chance of fertilization if an ovum is available:

$$
t=\frac{1}{\mathrm{k}_{N}} \cdot \ln \left(\frac{N_{o}}{N_{i}}\right)=\frac{\mathrm{t}_{1 / 2}(N)}{\ln 2} \cdot \ln \left(\frac{N_{o}}{N_{i}}\right)
$$

This equation provides a parameter for semen quality, since $F=f(t)$. To make it fully effective, the shape of this function must be known. We have to consider the chance that fertilization will occur at or before $t$, if during the period $t-t_{0}\left(t_{o}\right.$ is the moment when the spermatozoa are shed) increasing numbers of spermatozoa become available at $p$, either waiting for the ovum to appear, or approaching an ovum that is already present. Obviously, if the ovum is produced randomly, the probability that fertilization will occur, increases with time.

Since $F$ increases asymptotically to a limiting value $\left(F_{\infty}\right), t$ is found to be proportional to $\ln F$. Accordingly we get a linear relationship between the logarithm of the expected fertility $\mathrm{E}(F)$ and $t_{1 / 2}(N) \cdot \log \left(N_{o} / N_{i}\right)$. If $N_{i}$ is small as compared to $N_{o}$, $\log \left(N_{o} / N_{i}\right)$ will approach to $\log N_{o}$, since $N_{i}$ has an absolute lower limit of 1 . Consequently, if no data are available permitting to estimate $N_{i}$, the fertility of semen may be evaluated on a logarithmic scale by $t_{1 / 2}(N \bar{v}) \cdot \log (N \bar{v}) 。$ if motility is taken into account, or by $t_{1 / 2}(N) . \log N_{0}$ if motility is considered constant, as when comparing different concentrations to be used in artificial insemination with the same semen sample.

The slope constant of the $\log F=f\left[\mathrm{t}_{1 / 2}(N \vec{v}) \cdot \log (N \bar{v})\right]$ plot is now $1 / \mathrm{t}_{1 / 2}(F) . \mathrm{t}_{1 / 2}(F)$ is defined as the period of time in which the increment of the probability of fertilization falls to half its original value, if spermatozoa are supplied at a constant rate; in fact it is a measure for the overall efficiency of the fertilization process. The higher its reciprocal value, $1 / t_{1 / 2}(F)$, the higher the efficiency of increasing numbers of spermatozoa will be, or in other words, the more sensitive the female population is with respect to sperm numbers. It may be expected to vary with external conditions if these provide a bias with respect to the random distribution of the distance $\Delta(p, t)$ that must be traversed from the site of shedding of the spermatozoa to the meeting place with an ovum.

However, all constants required by the theory are amenable for experimental evaluation for a given sire with respect to a given population of females under any standard system of management.

\section{Verification of the theory with experimental data}

For experimental checking of the preceding derivations, fertilization rates resulting from the use of different dilutions from every ejaculate are needed. Such experimental results are available in the literature (Branton, Kellgren and Patrick, 1953; Willett and Larson, 1952; TANeja and Gowe, 1962) and have been analysed for the present purpose.

A straight-line transformation of eq. (6) is obtained by plotting $\log F_{\infty} /\left(F_{\infty} \rightarrow F\right)$ vs $\log N_{o}$, from which the constants $\mathrm{K}=\mathrm{k}_{F} / \mathrm{k}_{N}$ (slope constant) and $N_{i}$ (the intersection with the abscissa) can be evaluated. Of course, all constants can be 
evaluated by functional analysis of all available $x, y$ values, but a best estimate of $F_{\infty}$, which is important to get for the best possible fit of the linear regression line to the transformed data, can be obtained in a simple way by applying a graphical method derived for similar functions by HEYDECKER (1925), which is shown in FIG. 5 .

Good agreement between the field results obtained with different dilutions of 451 bull ejaculates, used in 72,336 inseminations, published by WILLETT and LARSON (1952) was obtained, as shown by FIG. 1 and 2 , as well as with their other experimental results concerning 236 ejaculates and 10,745 inseminations. The ratio $\mathbf{k}_{F} / \mathbf{k}_{N}=$ $\mathrm{t}_{1 / 2}(N) / \mathrm{t}_{1 / 2}(F)$ calculated from their data was 1.7 .

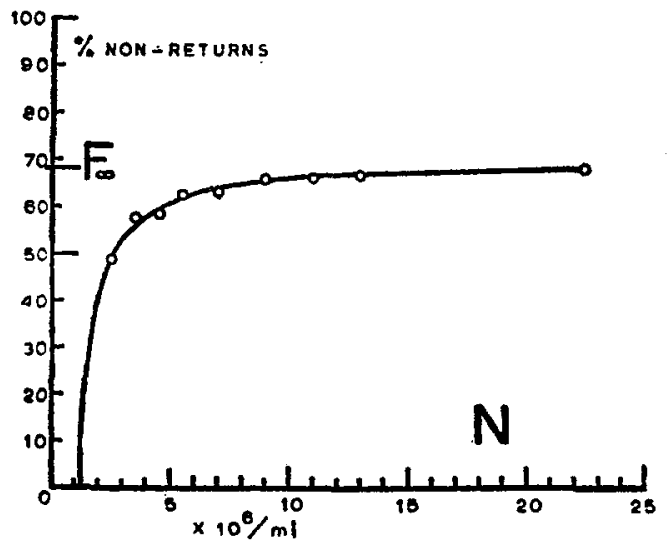

FIG. 1

Functional relationship between fertility (expressed as the percentage of non-returns after first insemination) and sperm numbers $N$, calculated from numerical data by WILLETT and LARSON (1952) by applying equations 5-7. The experimental results have been taken from WILLETT and LARSON's Table 4, based on 72,336 inseminations from 451 bull ejaculates.

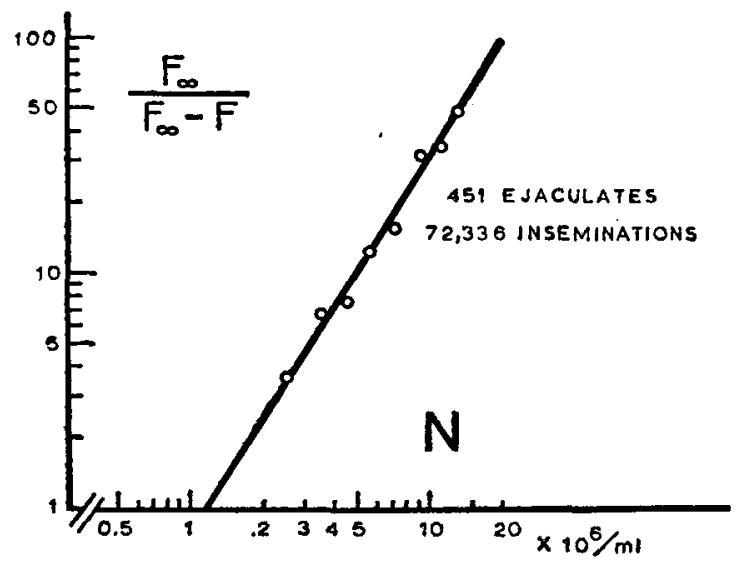

FIG. 2

Straight-line transformation of the results given in FIG. 1, according to equation (5), showing good agreement between theory and experiment. Experimental results of WILLETT and LARSON.

Good agreement is also obtained with the results by Branton, Kellgren and Patrick (1953), giving $F$ in relation to the product of sperm number $\times$ visual "percentage motility" rating, based on 8,356 inseminations from 275 bull ejaculates (FIG. 3). Although the multiplication of accurate relative sperm numbers by inaccurate "motility" estimations has spoiled much of the overall accuracy, the correlation coefficient of the transformed data is still high: $\mathrm{r}=+0.93, \mathrm{P}<0.001$. This is much higher 
than the erroneous linear correlation calculated by these authors themselves, which was "r" $=+0.219, \mathrm{P}<0.01$. The ratio $\mathrm{t}_{1 / 2}(\mathrm{Nm}) / \mathrm{t}_{\frac{1}{2}}(F)$ calculated from these data was 1.8 .

The experimental results of inseminating hens with various doses of undiluted semen, published by TANEJA and Gowe $(1961,1962)$ were also found to be in agreement with the theory, the correlation coefficient of the transformed data of three strains of fowl taken together being $\mathrm{r}=0.96, \mathrm{P}<0.001$ (van DuIJ, $1964 \mathrm{a}$, e). The agreement with these experimental results is especially important, because these data extend over the whole range of zero fertility up to the region where a limiting value is approached, whereas in cattle no reliable data with respect to very low sperm numbers are available (FIG. 4).

Whereas functional analysis of the empirical results yields the same general equation as the kinetic theory derived in this paper, the empirical constants cannot be interpreted correctly as the quotient of two rate constants $\left(\mathrm{k}_{F} / \mathrm{k}_{N}\right.$, or $\mathrm{k}_{F} / \mathrm{k}_{N}$, respectively) without the theoretical foundation of the equations.

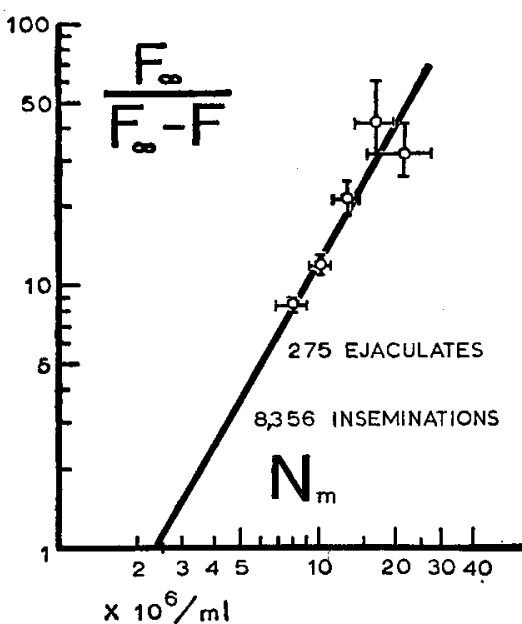

FIG. 3

Straight-line transformation of fertility data as a function of the product of sperm number $X$ visual "\% motility" rating, given in TABLE 1 , by BRANTON, KELLGREN and PATRICK (1953), based on 8,356 inseminations from 275 bull ejaculates. The agreement is good, even where the multiplication of accurate sperm numbers by inaccurate "motility" estimations has spoiled much of the overall accuracy. The goodness of fit is shown by the correlation coefficient for the straight-line data $r=+0.93, P<$ 0.001 . The indicated estimations of error have been calculated on the assumption of a Poisson distribution of sampling errors. The result invalidates the interpretation, given to the experimental results by the original authors, obtained by neglecting the fundamental boundary condition: $\mathrm{x} \geqq 0, \mathrm{y}=0$, i.e. at sperm concentration 0 , fertility must be 0 , whereas their regression indicates about $50 \%$ fertility at $\mathrm{Nm}=0$.

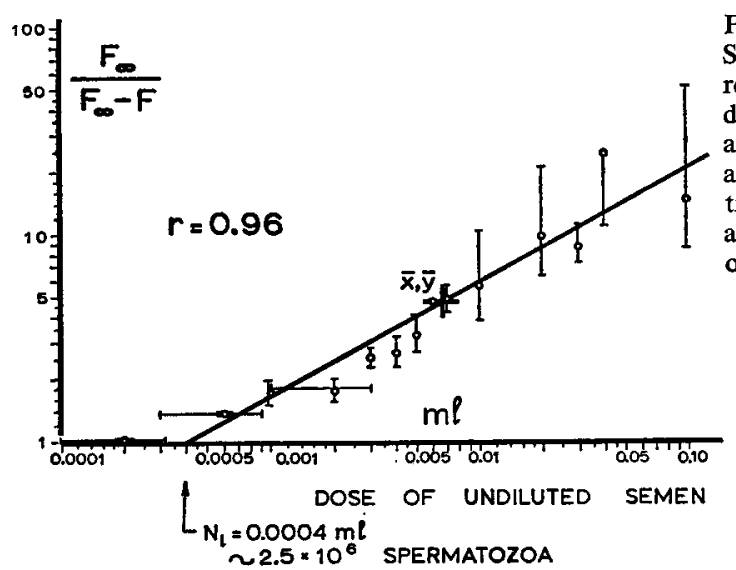

Neth. J. agric. Sci., Vol. 13 (1965) No. 4 (November)
FIG. 4

Straight-line transformation of insemination results obtained with varying doses of undiluted semen in fowl, published by TANEJA and GOWE (1962). Fertility $F$ is expressed as the number of days following insemination until the last fertile egg was laid. Averages of the data obtained with three strains of fowl. $\mathrm{r}=0.96, \mathrm{P}<0.001$. 
For estimating the adequacy of $t_{1 / 2}(N \bar{v}) \cdot \log (N \bar{v})$ as a quality index for the fertility of semen so far as this depends on sperm numbers and their movement characteristics, the results of photo-electric measurements of ejaculates from 40 different bulls, chosen randomly during several years, have been evaluated in this way and compared with the results obtained in A.I. practice in the same province (Utrecht, Netherlands) with semen from regular A.I. bulls of Dutch-Friesian and Black white-faced breeds (Jaarverslag K.I. 1957, '58, '59), the intercorrelation factor being season (FIG. 6). There is a
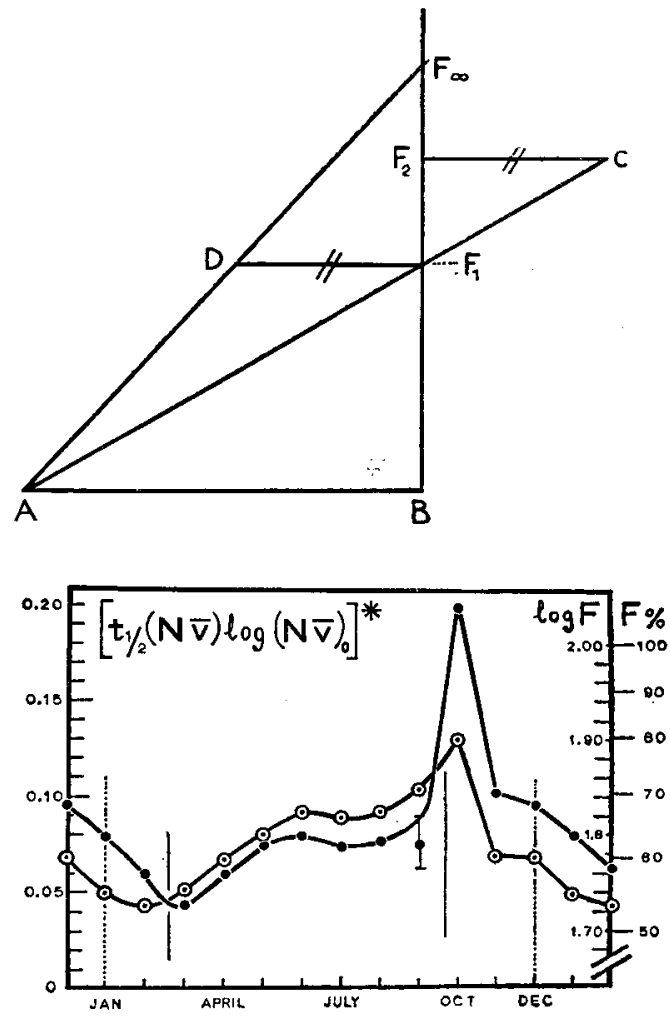

FIG. 5

Graphical determination of $F_{\infty}$ by application of HEYDECKER's method. On a base line $A B$, representing the scale unit, a perpendicular is erected, on which in the same units the fertilities $F_{1}$ and $F_{2}$ are set out, that correspond to two dilutions of the same ejaculate where $N_{1}: N_{2}=1: 2$. From $F_{2}$ draw a line parallel to the base, pointing to the right, and from $F_{1}$ draw a line parallel to the base, pointing to the left. Connect $A$ with $F_{1}$ and proceed till it intersects the $F_{2}$ horizontal at $C$. Measure $F_{2} C$ with compasses and transfer it to $F_{1} D$. Now connect $A$ with $D$ and prolong it till it intersects the perpendicular, where at the intersection, $F_{\infty}$ is obtained in the scale units chosen.

FIG. 6

General agreement between $t_{1 / 2}(N v) \log$ $(N \bar{v})_{0}$ and $\log$ Fertility $(\log F)$ in relation to season. The black circles indicate the first-mentioned relationship, the white dotted circles indicate the fertility function at the right-hand scales. The correspondence between the trends of both curves is highly significant ( $P \leqq 0.004$, binomial test).

The relative differences in level correspond to known bias with respect to the cow material, as explained in the text.

The $\mathrm{t}_{1 / 2}(N \bar{v}) \log (N \bar{v})_{0}$ values have been normalised to make $\underset{\operatorname{Jan}}{f} \mathrm{f}(x) \mathrm{d} x=1$. The fertility curve has been based on the results of 184,310 inseminations during a 4 years period.

striking and significant $(P \leqslant 0.004)$ similarity between the curves for fertility and that for semen quality computed in the way discussed here, whereas there is no similarity or any correlation between the fertility curve (based on results of 184,310 inseminations) and (i) visual "motility" estimates, alleged to represent "percentage of motile sperms" (FIG. 7); (ii) numbers of spermatozoa moving normally, per unit volume, or percentages of total sperm number that are moving normally, determined by photoelectric methods (trend indicated by the dotted line in FIG. 7); (iii) initial mean velocity of the spermatozoa determined by RiKMENSPOEL's (1957) photo-electric method (FIG. 8). Therefore it may be concluded that the present criterion is a more valid 


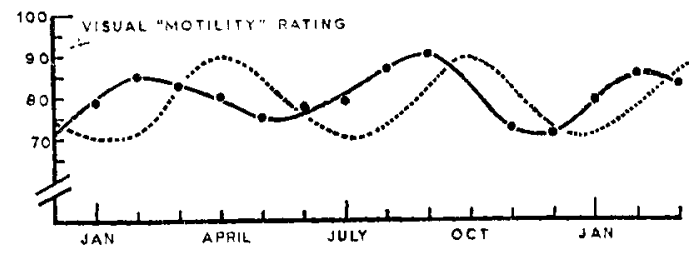

FIG. 7

Seasonal variation of mean visual "percentage motility" estimations of bull spermatozoa (full line), compared with the trend line of real percentages of motile spermatozoa, determined by physical methods (dotted line), demonstrating lack of correlation between visual estimations and objective measurements, whereas comparison with Fig. 5 shows that none of these correlates with fertility.

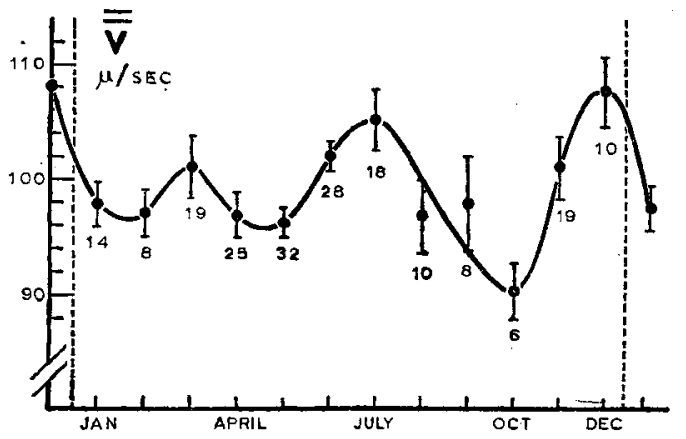

FIG. 8

Monthly averages of the initial mean velocities of bull spermatozoa $\bar{v}$, showing lack of correlation with the fertility data of Fig. 5 as well as lack of correlation with visual "\% motility" estimations (FIG. 6). For each month the number of ejaculates investigated is indicated.

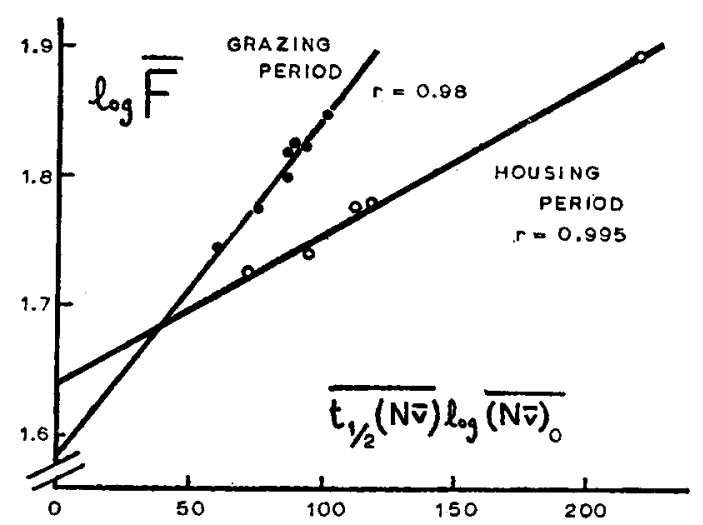

FIG. 9

Relationship between the logarithm of the mean percentage of fertilizations obtained with 184,310 inseminations of dairy cows, and the mean values of $t / \frac{1}{2}(N \bar{v}) \cdot \log (N \bar{v})$ (in arbitrary units) used as the criterion for evaluating seminal fertility according to the kinetic theory presented in this paper.

one for estimating semen quality, so far as fertility depends on sperm numbers and movement characteristics, than the other ones mentioned.

The remaining relative changes of level between the fertility and $t_{1 / 2}(N \bar{v}) \cdot \log (N \bar{v})$ curves coincides with known changes in the cow material, due to specific herd management in the Netherlands in general, and in the province of Utrecht in particular. During the housing season the percentage of successful inseminations is lowered because under housing conditions it is more difficult to establish the right moment for insemination when cows come into heat than when cows are in pasture. This lowered efficiency covers the period from mid-October till March, in the present material. A further effect is produced by biased selection of cows, especially in October and adjoining months, because the farmers prefer not to have calves during the grazing season; in consequence in October the main population of cows that come for in- 
semination will be of lower fertility level; notwithstanding this bias, the October peak of overall fertilization results is still there. I am indebted to Mr. J. DE RooIJ (1962) for providing these practical explanations for the remaining differences between the two curves. Because of these factors the results obtained in the period of March up to and including September (indicated as "grazing season") have been treated separately from results obtained during the other period ("housing season"). In both cases there was a strictly linear relationship as required by the kinetic theory (FIG. 9), with correlation coefficients of $r=+0.98$ and $r=0.995$ for the grazing and housing periods, respectively. These correlations are highly significant, $\mathrm{P} \ll 0.001$.

Further confirmative evidence has been obtained by analysis of similar data covering the period of March till December 1962, where the trend correlation between the two curves was $1.000, \mathrm{P}<0.002$, and the correlation $\log F \times \mathrm{t}_{1 / 2}(N \bar{v} \cdot \log (N \bar{v})$ : $\mathbf{r}=0.91, \mathbf{P}<0.001$ (van DuiJn, 1963, 1964b).

The difference in slope between the curves for the grazing and the housing periods is significant; since slope represents the constant $1 / t_{1 / 2}(F)$ according to eq. (12) this confirms the validity of the assumption that this parameter may be mainly governed by the female. The difference in height between the intersections of the curves given in FIG. 9 with the ordinate is not significant, i.e. the additive constant, containing $\frac{\mathrm{t}_{1 / 2}(N \bar{v})}{\mathrm{t}_{1 / 2}(F)} \cdot \log (N \bar{v})$ and any proportionality constant dependent on the unit system employed, might be independent of season. This requires further investigations.

How much better the correspondence of the criterion $t_{1 / 2}(N \bar{v}) . \log (N \bar{v})_{o}$ is for estimating $\log F$ than the separate components, is demonstrated by comparing these simpler correlations, calculated for the same material, none of which was found significant:

$\begin{array}{lcc} & \begin{array}{c}\text { Housing } \\ \text { period }:\end{array} & \begin{array}{c}\text { Grazing } \\ \text { period }\end{array} \\ \text { Corr. } \log (N \bar{v})_{0} \times \log F: & \mathbf{r}=0.56 & \mathbf{r}=0.11 \\ \text { Corr. } \mathrm{t}_{\frac{1}{2}}(N \bar{v}) \times \log F: & 0.40>\mathrm{P}>0.20 & \mathrm{r}=0.55 \\ \text { Corr. } \mathrm{t}_{\frac{1}{2}}(N \bar{v}) \cdot \log (N \bar{v})_{0}: & \mathbf{r}=0.84 & 0.20>\mathrm{P}>0.10 \\ & 0.10>\mathrm{P}>0.05 & \mathrm{r}=0.98 \\ & \mathrm{r}=0.995 & \mathrm{P}<10^{-4}\end{array}$

\section{Discussion and conclusions}

Although verification of the theory thus far has been obtained only with experimental results obtained in A.I. breeding of cattle and fowl, there is no reason why it should not be universally valid, since no specific presumption whatsoever has been made in the derivation. The theory can account for all known effects, since all other factors than movement parameters affecting the ability of spermatozoa to fertilize an ovum will be accounted for by $N_{i}$; if a large number of the available spermatozoa are in some way defective, $N_{i}$ will become large and $(N \vec{v})_{i}$ will also be large. Similarly, the limiting value $F_{\infty}$ may also be expressed as a function of other (at present unknown) factors of both male and female, if required. 
The theory is in agreement with the practical experience that large numbers of spermatozoa $\left(N_{i}\right)$ are required at the site of deposition in order to get a reasonable chance of fertilization. It further explains why there is bad correlation between velocity at some moment and fertility: the effect of longevity as measured by $t_{1 / 2}(N \bar{v})$ appears as a power of $(N \bar{v})_{0}$ and will therefore obscure the individual effect of velocity. On the other hand, it has been shown that there is a strong correlation between the rate of deterioration of spermatozoa and their mean velocity (VAN DUIJN, 1962). The higher the initial velocity, the shorter the period during which it is maintained and the shorter the period of survival of normally moving spermatozoa: Corr. - $\partial \bar{v} / \partial t$ $\times \bar{v}_{o}: \mathbf{r}=+0.57, \mathbf{P}<0.001$ (VAN DuIJn, 1962), $\mathrm{r}=0.85, \mathrm{P}<0.001$ (VAN DuiJn, $1964 \mathrm{~d}$ ) ; Corr. $N_{\text {moving }} \times \bar{v}_{o}: \mathrm{r}=-0.54, \mathrm{P}<0.001$ (van DuiJn, 1962), $\mathrm{r}=-0.57$, $\mathrm{P} \ll 0.001$ (van DuIJN, 1964a, d); Corr. $\log (-\partial \bar{v} / \partial t) \times \log \mathrm{t}_{1 / 2}(N): \mathrm{r}=-0.40$, $0.01>\mathrm{P}>0.001$ (VAN DuIN, 1962), $\mathrm{r}=-0.85, \mathrm{P}<0.001$ (van DuIJ, $1964 \mathrm{a}, \mathrm{d}$ ); Corr. $\log (-\partial v / \partial t) \times \log \mathrm{t}_{1 / 2}(N \vec{v}): \mathrm{r}=-0.87$ (van DuIJN, 1964d). The higher correlation coefficients in the most recent material mentioned have been obtained from more extensive data (degrees of freedom in each case 170 or more) gathered with higher measuring accuracy.

From these correlations it has already been concluded that the rates of velocity decrease with time, and the half-life period of the number of spermatozoa moving normally are more important for evaluating the quality of semen samples with respect to motility characteristics than the initial swimming velocity of the spermatozoa.

On the other hand, the present theory provides a criterion which can be determined even more easily. Since the parameters $N$ and $\vec{v}$ appear always as a product which is proportional to the number of passages across a certain spot in the field of a microscope per unit time, it is only necessary to determine this number of passages $n$ under standardized conditions and to repeat this at time intervals, for obtaining the information for calculating $\mathrm{t}_{1 / 2}(N \bar{v}) \cdot \log (N \bar{v})$. This can be done by photo-electric methods (RIKMENSPOEL, 1957; VAN DUIJN, 1960, 1961), but also visually, using a microchamber of standard dimensions (a depth of $40 \mu$ is required to provide unhampered movement) and a microscope equipped with a colour screen with a central hole of such a diameter that it is just filled by the image of a sperm head. A manually operated counter and a signal clock are the only other apparatus then required (VAN DUIJN, $1964 \mathrm{a}, \mathrm{c})$.

One objection may be put forward to the first assumption used in deriving the theory. The decrease of numbers of viable spermatozoa cannot remain truly exponential all time, because this would mean that the number would only become zero at infinite time, whereas in reality the last specimen will become immotile at an indefinite, but finite time (VAN DuIN, 1961, 1962). It may therefore be expected that the exponential relationship would in reality be part of a sigmoidal function. However, if it is assumed as a first approximation that fertilizing power is well maintained during the exponential part of the function, but would be negligible during the period where it may be expected to dip to zero, no refinement of the theory is required.

This latter condition seems to be fulfilled, because $N_{i}$ is found to be rather large so that $N$ will have dropped to $N_{i}$ before any deviation from the truly exponential function becomes apparent, and the same argument then follows for $N \bar{v}$ with respect to $\left(N \vec{v}_{i}\right.$. Since at $N_{i}$ and $\left(N \vec{v}_{i}, F\right.$ becomes zero, it is obviously of no importance 
how any further decrease takes place. The good agreement between the experimental results shews that this reasoning is sound. Of course, refinements could be made in due course if future results made them necessary.

\section{ACKNOWLEDGEMENT}

The author is indebted to Lord RothschiLD, F.R.S., Sc.D., for his stimulating interest and advice on the way of presentation of the fundamentals of the theory and for reading the manuscript.

\section{ANDERSON, J.}

1945 The semen of animals and its use for artificial insemination. Imp. Bur. Anim. and Breed. Genetics, Edinburgh.

Branton, C., C. B. James, 1951 T. E. PATrick and M. H. NewSOM

Branton, C., H. C. Kell- 1953 GREN and T. E. PATricK

Bratton, R. W., R. H. FOOTE and C. R. HENDERSON DuinN Jnr., C. vaN

The relationship between certain semen quality tests and fertility and the interrelationship of these tests. J. Dairy Sci. 34. $310-316$.

The importance of numbers of spermatozoa in relation to semen quality and fertility of bulls. J. Dairy Sci. 36, 13011307.

1954 The relationship between fertility and the number of spermatozoa inseminated. J. Dairy Sci. 37, 1353-1356.

1960 The effect of mixing ejaculates from different bulls on motil-

\section{REFERENCES} ity characteristics and acid production of the spermatozoa in a constant environment. J. Agric. Sci. 55, 69-74.

1961a Effects of light and optical sensitization by Acridine-orange on living bull spermatozoa. Nature (Lond.) 189, 76-78.

1961b Photodynamic effects of vital staining with diazine green (Janus green) on living bull spermatozoa. Exptl. Cell Research $25,120-130$.

1962a Toxic and photodynamic effects of toluidine blue on living bull spermatozoa. Exptl. Cell Research 26, 373-381.

$1962 \mathrm{~b}$ Velocity characteristics and numbers of bull spermatozoa in relation to ageing, determined by photo-electric methods. $J$. Reprod. Fertil. 4, 277-290.

1963 Bevruchtend vermogen van spermatozoa in verband met hun beweeglijkheidskenmerken en overlevingsduur. I. Kinetische theorie van de bevruchtingskans. Research Inst. Animal Husbandry "Schoonoord", Zeist, Netherlands. Report No. B 48.

1964a Het verband tussen aantal, beweeglijkheid en overlevingsduur van spermatozoa en de bevruchtingskans. Ned. Tijdschr. Geneesk. 108, 1116-1123.

1964b Kinetics of fertility of semen. Proc. Vth Intern. Congr. Anim. Reprod., Trento. 6-13 Sept. 1964, Vol. 4, 313-322.

$1964 \mathrm{c}$ A rational method for estimating fertility of spermatozoa in vitro. Proc. Vth Intern. Congr. Anim. Reprod., Trento 1964. Vol. 4, 323-328.

1964d Parameters of spermatozoan movement in relation to season, of bulls in the Netherlands. Proc. Vth Intern. Congr. Anim. Reprod., Trento 1964. Vol. 4, 307-312.

1964e Relationship between spermatozoan numbers and fertility. Intern. J. of Fertil. 9, 609-612. 
DuiJn Jnr., C. van, and R. RikMENSPOEL

EDgAR, D. G., and

S. A. AsDell.

FARRIS, E. J.

Herman, H. A., and E. W. Swanson

HEYDECKER, W. A.

Jaarversl. K.I.

Lasley, J. P., and

R. Bogart

MERCIER, E., and

G. W. Salisbury

Plisko, N. T.

RIKMENSPOEL, $\mathbf{R}$.
1960 The mean velocity and velocity distributions of normal bull spermatozoa at different hydrogen ion concentrations, derived from photo-electric measurements. J. Agric. Sci. 54, 300309.

1960 Spermatozoa in the female genital tract. J. Endocrinol. 2I, $321-326$.

1950 Human fertility and problems of the male. The Author's Press, Inc., Palisades Park, N. J., U.S.A.

1951 Variations in dairy bull semen with respect to its use in artificial insemination. Bull. Mo. Agric. Exp. Sta. No. 326.

1925 Résolution rapide de quelques problèmes usuels de sensitométrie. Sc. et Ind. Phot. $5,21 \mathrm{M}$.

Jaarversl. K.I. 1957, 1958, 1959, edited by Federatie K.I. Nederland, Velp, Netherlands.

1943 Some factors influencing reproductive efficiency of range cattle under artificial and natural breeding conditions. Res. Bull. Mo. Agric. Exp. Sta. No. 376.

1946 The effects of season on spermatogenic activity and fertility of dairy bulls used in artificial insemination. Cornell Vet. 36 , 301-311.

1961 Osemenenie svinei razlicnymi dozami spermy. Svinovodstvo. 15, 31-33. (Insemination of pigs with varying dosage of semen) Anim. Breed. Abstr. 30 (1962) No. 1189, p. 223).

1957 Photoelectric and cinematographic measurements of the motility of bull sperm cells. Thesis. Utrecht University, 1957.

1960 Measurements of the "motility" of bull sperm cells under various conditions. J. Agric. Sci. 54, 399-409.

and C. van DuIJ Jnr. 1960 Foto-electrisch en cinematografisch onderzoek van de beweeglijkheid van stierenspermatozoa. II. Foto-electrische metingen van enige eigenschappen van stierenspermatozoa. Tijdschr. Diergeneesk. 85, 1002-1014.

RIKMENSPOEL, R., and G. VAN HERPEN

RIKMENSPOEL, R., G. VAN Herpen, G. C. van Dam and $P$. EIJKHOUT

RoOH, J. DE

TANEJA, G. C., and R. S. Gowe

TAneja, G. C., and

R. S. Gowe
1957 Photoelectric and cinematographic measurements of the motility of bull sperm cells. Physics Med. Biol. 2, 54-63.

1960 Foto-electrisch en cinematografisch onderzoek van de beweeglijkheid van stierenspermatozoa. I. De meetmethodieken. Tijdschr. Diergeneesk. 85, 909-933.

1962 Personal communication.

1961 Effect of varying doses of undiluted semen on fertility in the domestic fowl. Nature (Lond.) 191, 828-829.

1962 Effect of varying doses of undiluted semen on fertility and hatchability in the domestic fowl. J. Reprod. Fertil. 4, 161-174. 